\title{
Experiencia con el uso de olaparib en pacientes con cáncer de ovario
}

\author{
Dolores Gallardo-Rincón, ${ }^{1,2 *}$ Gabriela Alamilla-García, ${ }^{1,2}$ Edgar Montes-Servín, ${ }^{2}$ Flavia Morales-Vázquez, ${ }^{1}$ \\ Claudia Cano-Blanco, ${ }^{1}$ Jaime Coronel-Martínez, ${ }^{1}$ Antonio Bahena-González, ${ }^{1,2}$ Raquel Gerson-Cwilich, ${ }^{4}$ \\ David Isla-Ortiz, ${ }^{3}$ Alfredo Toledo-Leyva, ${ }^{2}$ Elizabeth Montes-Servín, ${ }^{2}$ David Michel-Tello ${ }^{2}$ y \\ Raquel Espinosa-Romero',2 \\ 'Departamento de Oncología Médica; ${ }^{2}$ Programa de Cáncer de Ovario; ${ }^{3}$ Departamento de Ginecología Oncológica. Instituto Nacional de Cancerología; \\ ${ }^{4}$ Departamento de Oncología Médica, Centro de Cáncer, Centro Médico ABC. Ciudad de México, México
}

\section{Resumen}

Introducción: Más del $20 \%$ de los cánceres de ovario puede ser hereditario y la mayoría tiene mutaciones BRCA. El 33 \% de las pacientes mexicanas con mutación BRCA1 tiene la mutación fundadora, la cual es una deleción de los exones 9 al 12 (BRCA1 ex9-12del). Los tumores con mutaciones en BRCA son más sensibles a inhibidores PARP como olaparib. Objetivo: Mostrar la experiencia clínica del uso de olaparib en el Instituto Nacional de Cancerología de México. Método: Se estudiaron las pacientes con cáncer de ovario tratadas con olaparib de noviembre de 2016 a diciembre de 2018 y se describieron sus características, respuesta clínica, supervivencia libre de progresión y toxicidades. Resultados: Se evaluaron 19 pacientes, 78.9 \% presentó mutación BRCA1, del cual $21.1 \%$ era portador de la mutación fundadora ex9-12del. La mediana de supervivencia libre de progresión global fue de 12 meses, para las pacientes tratadas con olaparib de mantenimiento posterior a segunda y tercera línea fue superior a los 15 meses y para las de cuarta línea o más fue de 8.3 meses. Las pacientes con mutación fundadora presentaron mejores respuestas. Las toxicidades fueron similares a las de estudios con el uso de olaparib. Conclusiones: Olaparib ofrece mayor beneficio en supervivencia libre de progresión como tratamiento de mantenimiento después de la primera y segunda recaída. Las pacientes con mutación fundadora han tenido respuesta sostenida.

PALABRAS CLAVE: Mutación BRCA. Cáncer de ovario. Inhibidor PARP. Mutación fundadora de BRCA1. Olaparib.

\begin{abstract}
Introduction: More than the twenty percent of ovarian cancers are hereditary, and most have BRCA mutations. The $30 \%$ of Mexican patients with the BRCA1 mutation have the BRCA1 gene exon 9-12del deletion founder mutation (BRCA1 ex9-12del). BRCA-mutated tumors are more sensitive to PARP inhibitors such as olaparib. Objective: To show the clinical experience on the use of olaparib at Instituto Nacional de Cancerología in Mexico. Method: Ovarian cancer patients treated with olaparib from November 2016 to December 2018 were studied, and their characteristics, clinical response, progression-free survival (PFS) and toxicities were described. Results: Nineteen patients were assessed, with BRCA1 mutation being found in $78.9 \%$, out of which $21.1 \%$ were carriers of the ex9-12del founder mutation. The median of PFS was 12 months; for patients treated on second and third line it was greater than 15 months, and for those treated with a fourth and subsequent line it was 8.3 months. Patients with the founder mutation had better results. Toxicities were like those reported in previous studies. Conclusions: Olaparib offers greater PFS benefit as maintenance therapy after a first and second relapse. Patients with founder mutation have had sustained PFS.
\end{abstract}

KEY WORDS: BRCA mutation. Ovarian cancer. PARP inhibitor. BRCA1 founder mutation. Olaparib.

Correspondencia:

*Dolores Gallardo-Rincón

E-mail: dgallardorincon@gmail.com
Fecha de recepción: 29-08-2019

Fecha de aceptación: 03-09-2019

DOI: 10.24875/GMM.19005494
Gac Med Mex. 2019;155:585-589

Disponible en PubMed

www.gacetamedicademexico.com 


\section{Introducción}

El cáncer de ovario es el tipo de cáncer ginecológico que presenta mayor mortalidad. La incidencia anual a nivel mundial estimada para 2018 fue de 295414 nuevos casos, con 184799 muertes, lo que correspondió a $63 \%$. En cambio, el cáncer de mama presentó una incidencia superior a los 2 millones de nuevos casos, con una mortalidad de $30 \%$, mientras que la incidencia del cáncer de cérvix fue de 569847 nuevos casos, con una mortalidad de $55 \%$. En México, la incidencia estimada para cáncer de ovario en 2018 fue de 4759 casos y 2765 muertes (58\%).

Más de $20 \%$ de los cánceres de ovario puede ser hereditario ${ }^{2}$ y en 10 a $15 \%$ de las mujeres caucásicas están asociados con las mutaciones de línea germinal en los genes BRCA. La prevalencia de las mutaciones en $B R C A 1 / 2$ es elevada en las pacientes de origen hispano, en un estudio se reportó $28 \%$ de prevalencia; de 65 a $70 \%$ de las mutaciones correspondieron a BRCA1 y de 30 a $35 \%$ a BRCA2. Además, $33 \%$ de las mutaciones en $B R C A$ correspondió a la mutación fundadora BRCA1 ex9-12del (mutación fundadora). ${ }^{3}$

Los genes BRCA están implicados en el proceso de reparación por recombinación homóloga de las rupturas de la doble cadena del ácido desoxiribonucleico (ADN). Las células neoplásicas con mutaciones en los genes BRCA tienen una capacidad limitada para reparar daños en el ADN, a pesar esto siguen siendo viables y sobreviven mediante el uso de vías alternas de reparación. Existe una familia de proteínas denominadas PARP (poli ADP ribosa polimerasa), entre ellas se encuentran las enzimas PARP-1, PARP2 y PARP-3, necesarias en la reparación de rupturas de cadena sencilla del ADN.

Un estudio demostró que los tumores con mutaciones en BRCA eran especialmente sensibles a los inhibidores de PARP, debido a que la mutación en el gen BRCA1 induce incremento en los niveles de un factor de transcripción llamado ETS1, que en condiciones de hipometilación se une con mayor avidez a la región promotora del gen PARP1, lo cual incrementa su expresión. La enzima PARP1 está implicada en la vía de reparación heteróloga, la cual se realiza por la unión de extremos no homólogos del ADN, lo que la hace altamente propensa a errores. Esta vía de reparación brinda a la célula maligna la viabilidad suficiente para continuar con la progresión de la enfermedad. ${ }^{4}$
El olaparib es un inhibidor de PARP que al evitar la reparación de las rupturas de cadena sencilla del ADN aunada a la deficiencia en la reparación por recombinación homóloga causa muerte celular. ${ }^{5}$ Está indicado como tratamiento de mantenimiento en pacientes con cáncer de ovario con mutación en los genes BRCA que presentan respuesta parcial o completa después de quimioterapia basada en platinos. ${ }^{6}$

Los estudios clínicos disponibles muestran que el uso de olaparib como tratamiento de mantenimiento ofrece beneficios respecto al placebo en cuanto a la supervivencia libre de progresión (SLP) en pacientes con cáncer de ovario recurrente. El Estudio 19, un estudio de fase 2, aleatorizado, doble ciego, mostró que olaparib después de una primera línea de quimioterapia basada en platinos ofrece beneficios significativos en la SLP respecto al placebo para las pacientes con carcinoma seroso de alto grado (8.4 versus 4.8 meses, hazard ratio $=0.35$, IC $95 \%=0.25-0.49$, $p<0.0001$ ), en especial las que presentan $B R C A$ mutado (11.2 versus 4.3 meses, hazard ratio $=0.18$, IC $95 \%=0.10-0.31, p<0.0001) .{ }^{7}$ Posteriormente, SOLO-2, estudio aleatorizado de fase 3 , confirmó los resultados del Estudio 19, al mostrar un aumento significativo de la SLP (19.1 versus 5.5 meses, hazard ratio $=0.30$, IC $95 \%=0.22-0.41, p<0.0001$ ) en pacientes con cáncer de ovario recurrente sensible a platino con mutación en BRCA1/2 tratadas con olaparib. ${ }^{8}$ Además, el tratamiento de mantenimiento con olaparib se ha asociado con respuesta de larga duración, superior a dos años. ${ }^{9}$

En este trabajo se presenta la experiencia clínica del uso de olaparib en mujeres con cáncer de ovario con mutaciones en BRCA en el Instituto Nacional de Cancerología de México.

\section{Método}

Se seleccionaron pacientes con cáncer de ovario, mediante evaluación genética para la detección de mutaciones en BRCA1/2, de noviembre de 2016 a diciembre de 2018. Una muestra de sangre periférica de cada una de las pacientes fue analizada mediante secuenciación de segunda generación. Los grandes rearreglos como la deleción BRCA1 ex9-12del fueron evaluados mediante la prueba de amplificación dependiente de ligación múltiple (Quest Diagnostics). Se registraron las características clínicas entre las que se incluyeron la edad, la proporción de pacientes con cáncer de mama y ovario, la etapa clínica al momento del diagnóstico y subtipos histológicos del cáncer de 


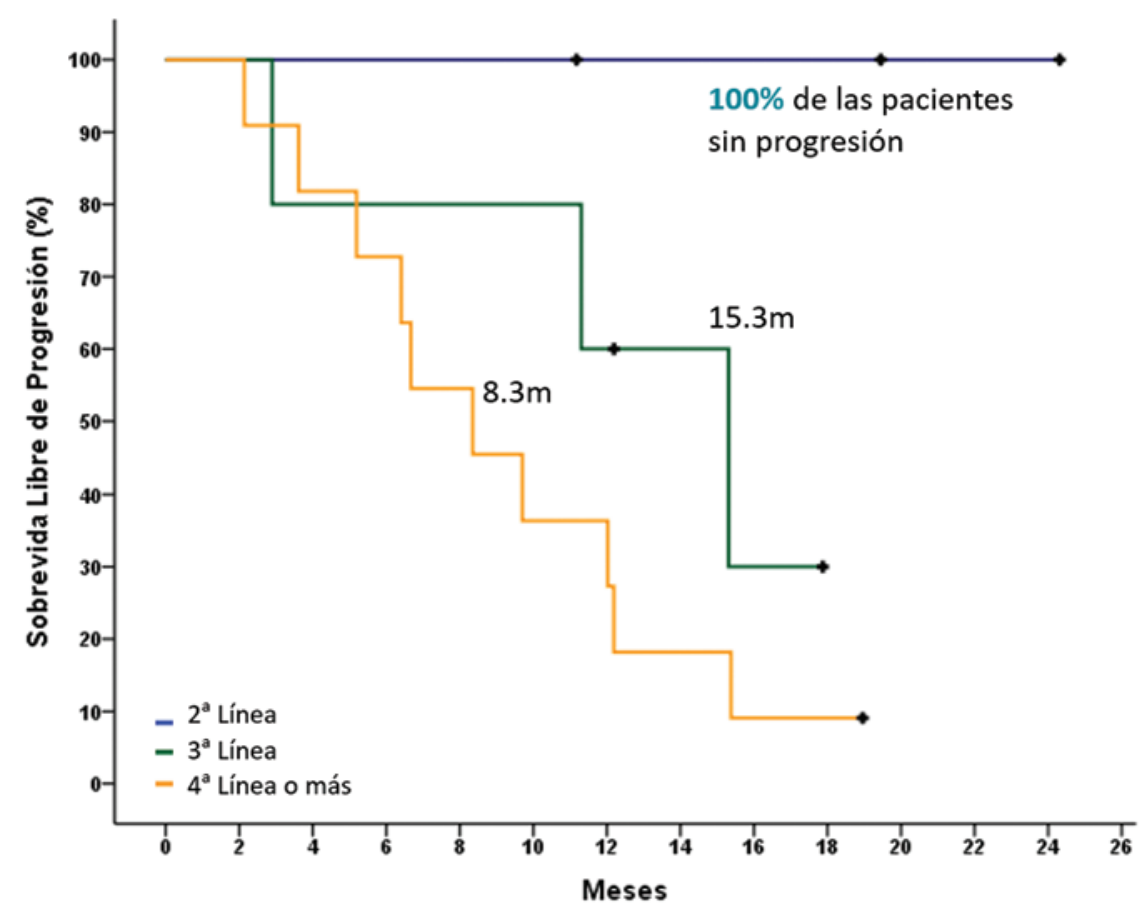

Figura 1. Supervivencia libre de progresión de las pacientes tratadas con olaparib de acuerdo con línea de tratamiento previo.

ovario. Todas las pacientes firmaron la carta de consentimiento informado para ser incluidas en el estudio.

Se registraron las líneas de tratamiento de quimioterapia previo a olaparib y su respuesta. Las pacientes fueron tratadas con $800 \mathrm{mg} / \mathrm{día}$ como terapia de mantenimiento después de una respuesta completa o una respuesta parcial a quimioterapia. De acuerdo con las toxicidades de grado 3 se realizó un ajuste a la mitad de la dosis (400 mg/día). Durante el tratamiento con olaparib se registró el estatus de sensibilidad a platino, líneas de tratamiento previo, SLP y ajuste de dosis por toxicidad relacionada con el tratamiento con olaparib.

El análisis de supervivencia se realizó por el método de Kaplan-Meier y las diferencias entre los grupos (segunda línea versus tercera línea, cuarta línea o más) se determinó por la prueba de log-rank o prueba de Breslow, considerando un valor $p<0.05$ como significativo. Todos los análisis se llevaron a cabo con el software SPSS versión 23 (SPSS, Inc., Chicago, IL, Estados Unidos).

\section{Resultados}

En el presente estudio se incluyó a 19 pacientes con cáncer de ovario, con mediana de edad de 51 años (rango de 40 a 67 años); $21.1 \%$ tuvo un diagnóstico de doble primario para cáncer de mama y ovario.
Al momento del diagnóstico, $10.5 \%$ de las pacientes presentaba cáncer en etapa clínica I, $5.3 \%$ etapa IIB, $10.5 \%$ etapa IIIB, $57.9 \%$ etapa IIIC y $15.8 \%$ etapa IV.

El $78.9 \%$ correspondió al subtipo histológico seroso de alto grado, $10.5 \%$ subtipo endometrioide y $10.5 \%$ adenocarcinoma sin determinar subtipo; $78.9 \%$ de la población presentó la mutación en BRCA1 y $21.1 \%$ en BRCA2; cuatro pacientes $(21.1 \%)$ tuvieron la mutación fundadora BRCA1 ex9-12del.

Las combinaciones de tratamiento previo a olaparib consistieron en carboplatino más paclitaxel o gemcitabina en $52.6 \%$ de las pacientes, cisplatino más paclitaxel o gemcitabina en $36.8 \%$ de los casos y carboplatino más paclitaxel más bevacizumab en $10.5 \%$. El resultado del tratamiento previo con olaparib fue respuesta completa en $42.1 \%$, respuesta parcial en $52.6 \%$ y enfermedad estable en $5.3 \% ; 47.4 \%$ fue parcialmente sensible al platino (intervalo libre de platino de seis a 12 meses) y $52.6 \%$ fue sensible al platino (intervalo libre de platino > 12 meses).

El $15.8 \%$ de las pacientes fue tratado con olaparib de mantenimiento posterior a segunda línea (después de la primera recaída), $26.3 \%$ posteriror a tercera línea y $57.9 \%$ posterior a cuarta línea o más. Hasta diciembre de 2018, $36.9 \%$ de las pacientes seguía recibiendo olaparib. Las cuatro pacientes con la mutación fundadora BRCA1 ex9-12del continuaban con respuesta al tratamiento de mantenimiento con olaparib. 
Tabla 1. Toxicidades relacionadas con el tratamiento con olaparib

\begin{tabular}{|c|c|c|c|c|c|c|}
\hline \multirow{2}{*}{ Característica } & \multicolumn{3}{|c|}{ Grado 1 } & \multicolumn{2}{c|}{ Grado 2 } & \multicolumn{2}{c|}{ Grado 3 } \\
\cline { 2 - 8 } & $\%$ & $n$ & $\%$ & $n$ & $\%$ & $n$ \\
\hline $\begin{array}{c}\text { Toxicidad hematológica } \\
\text { Anemia* }\end{array}$ & 21.1 & $4 / 19$ & 26.3 & $5 / 19$ & 10.5 & $2 / 19$ \\
\hline $\begin{array}{c}\text { Neutropenia } \\
\text { Trombocitopenia }\end{array}$ & 10.3 & $1 / 19$ & 21.1 & $4 / 19$ & - & - \\
\hline Leucopenia & 26.3 & $5 / 19$ & - & - & - & \\
Linfopenia & 26.3 & $5 / 19$ & - & - & 16.7 & $3 / 19$ \\
\hline
\end{tabular}

Toxicidad no hematológica

Náusea

Fatiga

Cefalea

Dolor abdominal

Neumonitis**

\begin{tabular}{|c|c|c|c|c|c|}
\hline 52.6 & $10 / 19$ & 15.8 & $3 / 19$ & - & - \\
68.4 & $13 / 19$ & 31.6 & $6 / 19$ & - & - \\
\hline 47.4 & $9 / 19$ & - & - & - & - \\
\hline 52.6 & $10 / 19$ & 15.8 & $3 / 19$ & - & - \\
- & - & - & - & 5.2 & $1 / 19$ \\
\hline
\end{tabular}

${ }^{*}$ Ajuste de dosis a $50 \%$ por anemia.

${ }^{*}$ Con antecedente de radioterapia por cáncer de mama.

La mediana de SLP global de las pacientes fue de 12 meses (IC $95 \%=8.74-15.31$ ). Las pacientes tratadas con olaparib posterior a segunda línea de tratamiento no progresaron después de 16 meses de tratamiento, mientras que la mediana de SLP para las pacientes tratadas con olaparib posterior a tercera línea fue de 15.3 meses (IC $95 \%=9.0-21.5$ ) y de 8.3 meses (IC $95 \%=4.5-11.8)$ para las tratadas con olaparib de cuarta línea o más, con una tendencia a una diferencia significativa en SLP entre estos dos últimos grupos de pacientes $(p=0.056)$ (Figura 1).

El tratamiento con olaparib se asoció con toxicidades hematológicas y no hematológicas, en la mayoría de los casos fueron grado 1, en 47.4 \% (9/19) se requirió reducción de dosis. La principal toxicidad hematológica fue anemia, que afectó a $58 \%$ de las pacientes, de las cuales $37 \%$ fue grado 2 o 3 y requirió ajuste de dosis en $50 \%$ en tres de las ocho pacientes que habían recibido cuatro o más líneas de tratamiento. Otras toxicidades hematológicas con menor frecuencia fueron neutropenia, trombocitopenia, leucopenia y linfopenia. La náusea fue la principal toxicidad entre las no hematológicas, afectó a $68 \%$ de las pacientes, $53 \%$ con grado 1. Otras toxicidades fueron fatiga, cefalea, dolor abdominal y neumonitis. Entre las toxicidades grado 1 se observó mayor proporción de las no hematológicas, mientras que entre las de grado 2 y 3 hubo mayor proporción de reacciones adversas hematológicas (Tabla 1).

\section{Discusión}

El estudio de cohorte realizado con las pacientes tratadas con olaparib en el Instituto Nacional de
Cancerología muestra un beneficio en la mediana de SLP de 12 meses, similar a los 11.2 meses observados en el Estudio 19 para el grupo de pacientes con mutación BRCA. ${ }^{7}$ El mayor beneficio en la SLP se observó en las pacientes tratadas con olaparib como terapia de mantenimiento después de la primera y la segunda recaída (> 15 meses), comparado con el grupo tratado después de tres o más recaídas (8.3 meses).

Los estudios previos no han documentado diferencias en la respuesta terapéutica entre pacientes con mutaciones $B R C A 1$ y $B R C A 2,{ }^{7,8}$ sin embargo, nuestra investigación mostró mejores resultados en las cuatro pacientes con mutación fundadora BRCA1 ex9-12del con SLP de hasta 18.9 meses. Estas cuatro pacientes representaron $21.1 \%$ de las pacientes con la mutación BRCA1.

Los datos de toxicidad fueron similares a los observados en el estudio SOLO2, con mayor incidencia de anemia en nuestro estudio asociada con mayor número de líneas de quimioterapia previa. ${ }^{8,10}$ Por otro lado, se identificó a una paciente con neumonitis que tuvo diagnóstico de cáncer de mama 15 años antes, tratada con radioterapia previa, lo que podría haber condicionado este efecto secundario con sinergia al uso de olaparib.

\section{Conclusiones}

El número de líneas previas es un factor pronóstico crucial para la respuesta a olaparib en terapia de mantenimiento que se traduce en una mejor SLP. Los datos de los estudios previos con olaparib y nuestros resultados en población de pacientes con cáncer de ovario recurrente sensible parecen indicar que el mejor escenario de tratamiento de mantenimiento con olaparib es posterior a terapia de segunda y tercera línea en comparación con pacientes que han recibido cuatro o más líneas previas.

La reducción de dosis de olaparib en pacientes con toxicidad hematológica o gastrointestinal sugiere no tener impacto negativo en la SLP.

La toxicidad estuvo asociada con mayor número de líneas de quimioterapia previa. Existe tendencia de SLP sostenida en terapia de mantenimiento con olaparib en pacientes con mutación fundadora, esta es la primera vez que se reporta dicho hallazgo.

Nuestros resultados apoyan el uso de olaparib como tratamiento de mantenimiento en pacientes con cáncer de ovario recurrente platino sensible, de manera ideal después de la primera y segunda recaída. 


\section{Conflicto de intereses}

Dolores Gallardo Rincón y Gabriela Alamilla García han participado como ponentes para AstraZeneca. Dolores Gallardo Rincón también ha participado como ponente con Roche y Lilly. Los otros autores no tienen conflictos de intereses que declarar.

\section{Bibliografía}

1. Ferlay J, Ervik M, Lam F, Colombet M, Mery L, Piñeros M, et al. Globa cancer observatory: cancer today. Francia: International Agency for Research on Cancer: 2018.

2. Toss A, Tomasello C, Razzaboni E, Contu G, Grandi G, Cagnacci A et al. Hereditary ovarian cancer: not only BRCA 1 and 2 genes. Biomed Res Int. 2015:2015:341723.

3. Villarreal-Garza C, Álvarez-Gómez RM, Pérez-Plasencia C, Herrera LA, Herzog J, Castillo D, et al. Significant clinical impact of recurrent BRCA1 and BRCA2 mutations in Mexico. Cancer. 2015;121:372-378.

4. Li D, Bi FF, Cao JM, Cao C, Li CY, Liu B, et al. Poly (ADP-ribose) polymerase 1 transcriptional regulation: a novel crosstalk between histone modification $\mathrm{H} 3 \mathrm{~K} 9 \mathrm{ac}$ and ETS1 motif hypomethylation in BRCA1-mutated ovarian cancer. Oncotarget. 2014;5:291-297.
5. Buisson R, Dion-Côté AM, Coulombe $\mathrm{Y}$, Launay H, Cai H, Stasiak AZ, et al. Cooperation of breast cancer proteins PALB2 and piccolo BRCA2 in stimulating homologous recombination. Nat Struct Mol Biol. 2010;17:1247-1254

6. FDA approved olaparib (LYNPARZA, AstraZeneca Pharmaceuticals LP) for the maintenance treatment of adult patients with deleterious or suspected deleterious germline or somatic BRCA-mutated (gBRCAm or sBRCAm) advanced epithelial ovarian, fallopian tube or primary peritoneal cancer who are in complete or partial response to first-line platinum-based [Sitio web]. EE. UU.: Food and Drug Administration; 2018.

7. Ledermann J, Harter P, Gourley C, Friedlander M, Vergote I, Rustin G, et al. Olaparib maintenance therapy in patients with platinum-sensitive relapsed serous ovarian cancer: a preplanned retrospective analysis of outcomes by BRCA status in a randomised phase 2 trial. Lancet Oncol. 2014;15:852-861.

8. Pujade-Lauraine E, Ledermann JA, Selle F, Gebski V, Penson RT, Oza AM, et al. Olaparib tablets as maintenance therapy in patients with platinum-sensitive, relapsed ovarian cancer and a BRCA1/2 mutation (SOLO2/ENGOT-Ov21): a double-blind, randomised, placebo-controlled, phase 3 trial. Lancet Oncol. 2017;18:1274-1284.

9. Lheureux S, Lai Z, Dougherty BA, Runswick S, Hodgson DR, Timms KM, et al. Long-term responders on olaparib maintenance in high-grade serous ovarian cancer: clinical and molecular characterization. Clin Cance Res. 2017;23:4086-4094.

10. Moore K, Colombo N, Scambia G, Kim B-G, Oaknin A, Friedlander M, et al. Maintenance olaparib in patients with newly diagnosed advanced ovarian cancer. N Engl J Med. 2018;379:2495-2505. 